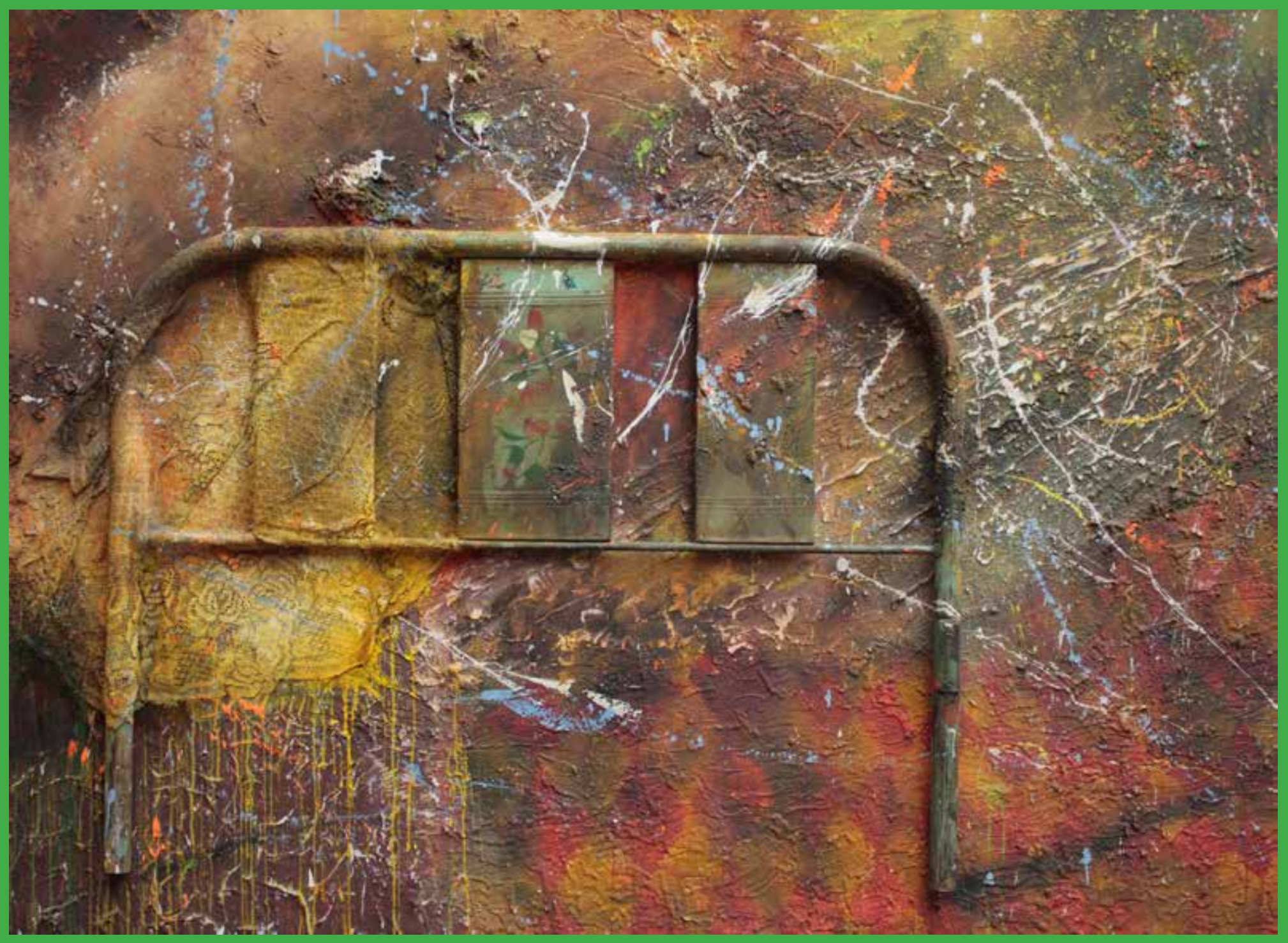

Sergio Giraldo / Discapacidad Física / "Ref. No 22" / Mixta (pigmentos minerales, brea, tela, objeto encontrado)

Hernando Augusto Clavijo Montoya. M.D., M.Ed., M.Sc.

Profesor del Departamento de Ciencias Básicas,

Escuela Colombiana de Rehabilitación. Bogotá, Colombia. haclavijo@gmail.com 


\title{
REFLEXIONES SOBRE LA INTEGRACIÓN DE LAS CIENCIAS BÁSICAS BIOMÉDICAS CON LA PRÁCTICA CLÍNICA EN PROGRAMAS DE PREGRADO
}

\section{Reflections on the integration of basic biomedical sciences with clinical practice in undergraduate programs}

Fecha de recepción: 16 de julio de 2013 - Fecha de aprobación: 17 de octubre de 2013

\begin{abstract}
RESUMEN
Las ciencias biomédicas han incorporado desde hace varios años, y de forma definitiva, los lenguajes de la genética, la biología molecular y la bioinformática con un volumen de publicaciones que cada año aumenta de forma exponencial. Sin embargo, tradicionalmente es reconocida la preocupación por la integración de los descubrimientos de las ciencias básicas biomédicas con la práctica clínica, la cual es de interés científico y académico a nivel de pregrado y de postgrado así como en la práctica profesional en las ciencias de la salud. La medicina traslacional tiene por objetivo hacer estos procesos de integración de una forma más efectiva. En el artículo son tratados diferentes temas de interés, relacionados con la medicina traslacional, en diferentes programas de la salud con énfasis en la educación en el pregrado.
\end{abstract}

PALABRAS CLAVES medicina traslacional, medicina traduccional, educación traslacional.

\begin{abstract}
Since many years ago, basic biomedical sciences have been incorporating language of genetics, molecular biology, and bio-computing topics by releasing a number of publications that has grown yearly. However, the fact of joining the biomedical basic science findings with the clinical practice is a known worry, which is of interest to scientists and academics at under and postgraduate courses and also very important at the professional health science practice. Translational medicine aims to make this integration processes more effective. Several topics of interest are covered over the article, these are related to translational medicine from different health fields focusing on undergraduate education.
\end{abstract}

KEYWORDS

Translational medicine, translational education.

«No existe una categoría en la ciencia a la que se le pueda dar el nombre de ciencia aplicada. Existen la ciencia y las aplicaciones de la ciencia, unidas entre sí como el fruto a su árbol».

-Louis Pasteur (1822-1895) 
de práctica educativa (presencial, virtual o mixta conocida como B-Learning) que son más eficaces en los diferentes procesos de enseñanza-aprendizaje de las neurociencias, reflexión que incluye naturalmente a todas las ciencias básicas biomédicas.

alemán, fue el fundador de la neuroanatomía moderna. Edinger tenía una combinación inusual de un cientíico profundo, un profesor brillante, un artista plástico y un gestor muy competente. Edinger hacía sus clases muy particulares a sus alumnos al dibujar simultáneamente las estructuras intrincadas del cerebro con la mano izquierda mientras escribía su leyenda con la derecha (Prithishkumar, 2011).

Desde los tiempos de Edinger han surgido nuevos retos para la enseñanza de las neurociencias que ejemplifican, en general, los retos en la educación de las ciencias básicas biomédicas. Históricamente han sido mucho los científicos cuyas contribuciones fueron sustanciales en el desarrollo de la neuroanatomía, entre estos, los trabajos realizados por Camilo Golgi (1843-1926) así como los aportes realizados por Santiago Ramón y Cajal (1852-1934) marcaron los cimientos de la neuroanatomía dividiendo para diversos autores las neurociencias en el antes y el después de estos aportes, los cuales siguen siendo utilizados aún en la actualidad (Jones, 2007).

Sin duda, desde el punto de vista educativo de las ciencias básicas biomédicas, con los avances de la biología molecular, entre los que se encuentran los hallazgos del proyecto del genoma humano, uno de los principales retos es lograr la integración en las diferentes asignaturas de los niveles molecular, celular, tisular, orgánico, sistémico, así como del individuo, de la población y de los ecosistemas en un contexto de competencias y de logros con sus respectivos indicadores en la salud y la enfermedad. A esta situación se suma el reto de lograr la integración de las ciencias básicas biomédicas con las disciplinas de las prácticas clínicas, reto que se encuentra claramente documentado desde hace varios años, como es el caso del artículo: "Integration of basic science with clinical training" (Lewis, 1956) y en años recientes, en el artículo: "Integration and timing of basic and clinical science education" (Bandiera, Boucher, Neville, Kuper, \& Hodges, 2013), por citar dos ejemplos.

Esta integración constituye una preocupación constante en los procesos enseñanza-aprendizaje, entre otros campos del conocimiento, en la anatomía, la fisiología (Tworek, Jamniczky, Jacob, \& Hallgrímsson, 2013) la neuroanatomía (Zinchuk, Flanagan, Tubridy, Miller, \& McCullough, 2010) y la genética (Mainous, Johnson, Chirina, \& Baker, 2013). De otra parte, el caso de la neuroanatomía también ejemplifica los retos que tienen las ciencias básicas biomédicas en relación con el uso de las Tecnologías de la Información y la Comunicación (TICs).

Es así como para muchos educadores, las disecciones de los cadáveres humanos, que se remontan en sus orígenes ampliamente documentados en la Grecia de Alejandro Magno con Herófilo de Alejandría (entre 350 y el 280 a. de C.), han representado los mayores aportes que se hayan realizado para la comprensión de la neuroanatomía (Moon, Andreas, \& Cohen, 2010) teniendo en la actualidad a nivel educativo el complemento, para algunos, y la alternativa, para otros, de los modelos anatómicos y de los ambientes de enseñanza virtuales, constituyendo este, otro de los retos sobre los tipos

\section{DESARROLLO DEL TEMA.}

A la medicina traslacional (en español se le conoce también como traduccional, traducida, traslativa y trasladada, entre otros términos) se le ha definido como la rama de la investigación médica que intenta conectar más directamente la investigación básica con el cuidado de los pacientes. Surgiendo también términos como la educación traslacional que hace referencia a aquella que vincula las ciencias básicas con las asignaturas clínicas, lo cual ha sido una aspiración secular en las escuelas de medicina (Lifshits, 2009).

El término "traslacional" aún requiere un mayor consenso en su interpretación. Autores como Hasselbart (2008), explican que este término es un anglicismo, y que el término más adecuado debería ser "traduccional" porque en inglés significa la traducción entre dos lenguajes distintos, el de las ciencias básicas y el de sus aplicaciones clínicas. Otros autores interpretan la palabra "traslacional" como la unión de los conceptos de traducción y de traslado desde las ciencias básicas hacia la práctica clínica con la interacción también de la epidemiología (Fuentes, 2009).

Han existido diferentes interpretaciones de las aplicaciones de los enfoques traslacionales y, por esta razón, se ha propuesto una identificación de las diferentes etapas del proceso traslacional con categorías que van de la $\mathrm{T}_{1}$ a la $\mathrm{T}_{4}$. Por ejemplo, T1 comprende los primeros estudios en humanos, los estudios de fase 1 y fase 2 y las pruebas de concepto; T2 incluye los estudios de fase 3, el análisis de la eficacia clínica y las guías clínicas; T3 se relaciona con la diseminación de la información, el compromiso con la comunidad, la investigación de los servicios de salud y los estudios de efectividad y $\mathrm{T}_{4}$ hace referencia a la prevención, las modificaciones en el comportamiento, en los estilos de vida y, en general, a las medidas de salud pública y al estudio del impacto de estas medidas, (Woolf, 2008; Kiefe, 2011).

La integración con la práctica clínica también es una preocupación constante de otros campos del conocimiento diferentes de las ciencias básicas. De hecho, se han agregado otras áreas del conocimiento a la investigación traslacional. Por ejemplo, para algunos autores la investigación traslacional surge como respuesta de la crisis del antiguo modelo que suponía una gran simplicidad en la generación de la evidencia científica en salud y su implementación en la vida real.

El paso intermedio era, principalmente, la generación de guías clínicas para la práctica de la medicina basada en evidencia. Dichas guías eran, a su vez, fuente de información para quienes tomaban las decisiones de la salud pública en un país, para la generación y la evaluación de las políticas sanitarias. Si bien este mecanismo ha prevalecido por varias décadas y aún se mantiene en muchos países, hoy se reconoce la naturaleza no-lineal del proceso que vincula de manera efectiva la síntesis de la evidencia científica a soluciones sustentables en salud a nivel poblacional. Dicho proceso es analizado por la inves- 
tigación traslacional y considera la evidencia de distintas fuentes y de diversa naturaleza, incluyendo, entre otros, los estudios observacionales y los de naturaleza cualitativa (Cabieses \& Espinoza, 2011).

Las ciencias biomédicas han incorporado desde hace varios años y de forma definitiva los lenguajes de la genética, la bioquímica, la biología molecular y la bioinformática con un volumen de publicaciones que cada año aumenta de forma exponencial. Sin embargo, se observa que los investigadores experimentales y los investigadores clínicos muchas veces no hablan el mismo idioma, tienen entornos profesionales y puntos de vista diferentes, lo cual se traduce en dificultades en la comprensión y en la comunicación desde el punto de vista multidisciplinar (Smeesters, Deghorain, \& Steer, 2011).

Por ejemplo, los hallazgos encontrados en el culminado proyecto del genoma humano plantean retos de cómo la cantidad de información que se ha originado será integrada a nivel investigativo y educativo. En el cáncer, por ejemplo, desde que se completó el proyecto genoma, uno de los principales objetivos ha sido implementar la investigación traslacional en todos sus niveles como algo multidisciplinario e interdisciplinario en donde todos los actores deben estar involucrados, trabajar a la par así como mantener una constante comunicación y es una de las áreas en las que este enfoque presenta mayores avances (Hasselbart, 2008).

Así mismo, los resultados del proyecto del genoma humano son insumos muy importantes que deben ser considerados en la comprensión de la neurobiología de las enfermedades mentales, sugiriendo una "reingeniería" de las áreas clínicas que se debe hacer a partir de un enfoque traslacional con las ciencias básicas (Ferrell, 2009). De hecho, la investigación genómica tiene un impacto cada vez mayor en la medicina clínica, por ejemplo, algunas enfermedades serán redefinidas y en algunas situaciones se seleccionarán las terapias adecuadas para los pacientes con base en su perfil molecular, algo de lo que desde hace algunos años se comienza a hablar para el cáncer, la diabetes mellitus y la hipertensión arterial, entre otras enfermedades (Gamulin, 2003).

El ritmo de los descubrimientos de las variantes en farmacogenética que son prometedoras a nivel terapéutico ha aumentado también de forma exponencial en los últimos años. Sin embargo, la aplicación de este conocimiento para los pacientes ha sido lenta y es uno de los objetivos que tiene la investigación traslacional en farmacogenética (Shuldiner y otros, 2013).

La generación del conocimiento no siempre considera de forma inmediata su aplicación práctica, asumiendo que finalmente encontrará su utilidad de manera natural (Lifshitz, 2009).

Existe una importante cantidad de conocimiento que se ha generado desde las ciencias básicas biomédicas que no se han traducido en beneficios tangibles para la salud, sin embargo, se estima que la comprensión de los mecanismos moleculares relacionados con la salud y la enfermedad nos llevarán del conocimiento del "hardware" al conocimiento del "software" de la vida, que conducirá también a la reclasificación de varias enfermedades y a un mejor conocimiento de las interacciones de los sistemas biológicos con el medio ambiente (Zerhouni, 2012).
Un ejemplo prometedor de la integración reciente de las ciencias básicas biomédicas con las clínicas se verifica en investigaciones en donde se observa que la estimulación cortical, la interacción interhemisférica y las interacciones farmacológicas, entre otros, pueden modular la plasticidad cortical después del evento cerebrovascular para la recuperación de los pacientes (Floel \& Cohen, 2006). Un componente importante del desarrollo y la maduración del sistema nervioso central (SNC) se produce a través de la interacción y la experiencia con el medio ambiente de un individuo. La experiencia ambiental moldea las sinapsis, las conexiones anatómicas y la estructura del cerebro durante el desarrollo.

La evidencia reciente indica que la experiencia del medio ambiente relacionada con la actividad neuronal influye en una sorprendentemente amplia cantidad de conexiones del cerebro y la remodelación estructural después de la lesión en el adulto. Se necesitan más estudios para dilucidar plenamente los sustratos moleculares de las lesiones y la plasticidad neuronal relacionada para entender cómo la compleja interacción entre las moléculas se relaciona con el cambio anatómico persistente en el SNC (Overman \& Carmichael, 2013).

En fonoaudiología también es claro que los aportes desde las ciencias básicas en estudios en animales, en la cognición humana y en la informática, son relevantes para la investigación traslacional en el tratamiento de las afasias (Raymer y otros, 2008). Por ejemplo, los tratamientos de las afasias como consecuencia de los eventos cerebrovasculares, también se han beneficiado recientemente de los "Insights" de la neurociencia y de la neuropsicología a través del conocimiento de la plasticidad neuronal (Berthier \& Pulvermüller, 2011).

El interés de la conexión entre las áreas básicas y clínicas se encuentra documentado también en fisioterapia, siendo igualmente pertinente el estudio de la neuroplasticidad en la neurorehabilitacion (Sullivan, 2010). Definitivamente, los enfoques traslacionales cada vez son más visibles en las publicaciones científicas no solamente en la medicina sino todas las áreas de la salud, por ejemplo, se encuentran aparte de los ya citados, de interés en la fisioterapia reportes de investigaciones traslacionales en: rehabilitación de la lordosis lumbar para el manejo del dolor (Diab \& Moustafa, 2012), bioingeniería en las lesiones medulares (Wilcox, Cadotte, \& Fehlings, 2012) y tecnologías emergentes y terapias innovadoras en neurorehabilitación (Ifejika-Jones \& Barrett, 2011), entre otros.

En la terapia respiratoria: estudios de nuevas tecnologías en nebulización en la fibrosis quística (McCormack, Southern, \& McNamara, 2012), investigaciones sobre la farmacoterapia y los músculos esqueléticos en la enfermedad pulmonar obstructiva crónica (Steiner, Roubenoff, Tal-Singer, \& Polkey, 2012) y uso de biomarcadores en la lesión pulmonar aguda (Proudfoot, Hind, \& Griffiths, 2011).

Lifshits (2009) indica que "en la medicina se ha manifestado una separación entre las áreas básicas y las clínicas, condición que se observa desde hace tiempo en los currículos universitarios, al menos en el modelo Flexneriano, en los que parece haber dos carreras diferentes, la que se cursa en la primera mitad destinada a las ciencias básicas y la de la segunda mitad relativa a las disciplinas clínicas. Los muchos intentos de integración no siempre han sido exitosos, 
y no deja de ser una preocupación permanente para la planificación curricular y la formación de un médico científico.

En muchas universidades los profesores de ciencias básicas ya no son médicos, sino profesionales de otras áreas como biología o química". Esta situación no parece ser diferente en otras disciplinas de la salud, como es el caso de la enfermería, y las que hacen parte de la rehabilitación como la fisioterapia, la fonoaudiología, la terapia ocupacional y la terapia respiratoria. Por ejemplo, desde la perspectiva de la terapia ocupacional, citando un ejemplo, se reconoce también que a pesar de la disponibilidad de la investigación innovadora de salud, existe una brecha entre el conocimiento generado por la investigación y la utilización de esos conocimientos en situaciones prácticas de la vida real (Clark, Park, \& Burke, 2013).

En enfermería también existe la preocupación en el sentido de que a pesar de la importante cantidad de investigación realizada, se presenta un vacío entre las investigaciones y el uso de los resultados para la atención de los pacientes (Padilha, 2011). Los estudiantes de pregrado tienen una percepción similar, un estudio realizado con 317 estudiantes de medicina de diferentes nacionalidades permitió verificar que existe una importante percepción sobre el distanciamiento entre la investigación básica y su aplicabilidad en los pacientes y es preocupante que muchos estudiantes no tengan un conocimiento completo sobre los conceptos y los procesos relacionados con la medicina traslacional. Paradójicamente, muchos de sus profesores y de sus mentores se dedican a la investigación pero no se han dado las oportunidades o los espacios de desarrollar estas competencias en los estudiantes (Burgoyne, O’Flynn, \& Boylan, 2010).

Diversas investigaciones realizadas han permitido verificar que muchos descubrimientos en ciencias básicas solamente se traducen en un impacto a nivel terapéutico o preventivo varios años después (Ioannidis, 2004). De 101 artículos publicados entre 1979 y 1983, en 6 revistas científicas de diferentes áreas de ciencias básicas, e identificados con posibilidades terapéuticas o preventivas, se observó que 27 de los mismos tuvieron al menos una publicación relacionada con un ensayo clínico aleatorizado. Diecinueve de estos tuvieron al menos una publicación con un ensayo clínico aleatorizado positivo, de los cuales, los descubrimientos originales de 5 estudios son usados actualmente con autorización para el uso clínico. Además, los artículos en ciencias básicas que no se traducen en una publicación en estudios en humanos 10 a 12 años después, es poco probable que continúen siendo investigados posteriormente (Contopoulos-Ioannidis, 2003).

Los estudios basados en los análisis de traducción del impacto de las ciencias básicas a nivel terapéutico o preventivo así como la metodología, de lo que es reconocido por algunos autores como el triángulo de la biomedicina, comprendiendo en cada esquina del triángulo: los estudios en células y moléculas, los estudios en animales y los estudios en humanos, son importantes en términos políticos para evaluar los resultados de las inversiones que se deben hacer para acelerar el impacto de las investigaciones en ciencias básicas (Weber, 2013).

De hecho, la existencia de revistas científicas surgidas en años recientes como el Journal of Translational Medicine (http://www.transla- tional-medicine.com), el Journal of Cardiovascular Translational Research (http://www.springer.com/medicine/cardiology/journal/12265), el Stem Cells Translational Medicine (http://stemcellstm.alphamedpress.org/), el Translational Psychiatry (http://www.nature.com/tp/index.html) y el Neurorehabilitation and Neural Repair, que es presentada como una revista científica de la ciencias traslacionales para investigadores y clínicos (http://www.sagepub.com/journals/Journal201625/manuscriptSubmission\#tabview=title), entre otros, confirman esta tendencia.

Así mismo, también han surgido asociaciones como la Association for Clinical and Translational Science (http://www.actscience.org/) y la European Society for Translational Medicine (http://www.eutranslationalmedicine.org/). Es pertinente reflexionar y comenzar a llegar a acuerdos sobre las competencias e indicadores de logro que deben ser considerados en los programas de las asignaturas biomédicas.

De una parte está la inquietud, completamente comprensible, de si todos los contenidos incluidos en los programas de las asignaturas en realidad están justificados así como el nivel de profundidad de los mismos con base en los campos de acción de las diferentes profesiones y en este sentido es recomendable considerar lo que realmente corresponde al aprendizaje significativo, siendo que el enfoque de competencias y de resultados en el aprendizaje ayuda a enfocar esta situación.

En relación con los contenidos, competencias e indicadores de logro que se desean desarrollar en las disciplinas básicas biomédicas se identifican las siguientes reflexiones en la elaboración y en el desarrollo de los programas académicos: ¿son relevantes para la comprensión de la salud y la enfermedad?, ¿se encuentran en un contexto de aplicación profesional para cada Facultad?, ¿se encuentran en un contexto de aprendizaje para la investigación?, ¿son relevantes a un nivel de pregrado o de postgrado?, ¿existen espacios para que los estudiantes puedan reconocer las circunstancias que han llevado a desarrollar enfoques traslacionales en ciencias de la salud?, ¿existen espacios para que los estudiantes puedan comprender cómo se aplican los enfoques traslacionales? ¿es fomentada la interdisciplinariedad desde las perspectivas traslacionales?, ¿hay asignaturas electivas que permitan profundizar sobre la medicina traslacional?, iexiste un diálogo constante de doble vía entre los docentes de las ciencias básicas biomédicas y los docentes de los campos prácticos para lograr una mejor integración vertical en el currículum?

Existen investigaciones que ya comienzan a documentar la necesidad y el impacto de los enfoques traslacionales en la educación en los programas de pregrado. Por ejemplo, en la Universidad de Carolina del Sur en los Estados Unidos, desde el primer año en la formación médica se integran las neurociencias básicas en contextos explícitos a nivel clínico.

Esta experiencias han contribuido, junto con otras acciones, a mejorar el nivel de satisfacción de los contenidos aprendidos por parte de los estudiantes $(43,9 \%$ a $81,8 \%$ ) aunque los resultados en los test de conocimiento han tenido un impacto moderado (Holden, Cooper, \& Wong, 2012). En el Reino Unido, la integración de la farmacología básica con la farmacología clínica tiene una historia de más de 40 años con resultados que no siempre han sido óptimos, se espera que los avances actuales en innovación y la traducción del 
conocimiento brinden nuevas oportunidades y con esto lograr una mayor sinergia que se debe fomentar en los programas de pregrado y de postgrado, entre otras herramientas, a través de los enfoques traslacionales (Buckingham, 2012).

En la actualidad, los cursos relacionados con la biología molecular hacen parte de prácticamente todos los programas de medicina y de las ciencias de la vida e históricamente muchos descubrimientos en la salud y la enfermedad han tenido como origen investigaciones en el área básica. Es deseable que este tipo de contribuciones con sus aplicaciones sean incorporados en los cursos de biología molecular y celular para disminuir la brecha entre las ciencias básicas y los campos aplicados (Sitaraman, 2012).

A través de un convenio, de estudiantes que viajaron de la Universidad de Riyadh (Reino de Arabia Saudita) para asistir a un curso de verano en el St. Boniface Hospital Research Centre en Winnipeg (Canadá), en donde se integran los temas básicos con los clínicos, una investigación permitió verificar proporciones de satisfacción en los estudiantes del $100 \%$ en los diferentes aspectos estudiados, entre los que se encuentran: la ventaja de este enfoque de integración, el reconocimiento del valor de estudiar más allá de los libros, menos preocupación y más confianza en el programa de pregrado, la comprensión de la importancia de la investigación y el reconocimiento de que es considerada como una aproximación "más fácil" para aprender sobre los avances de las investigaciones (Ramjiawan y otros, 2012).

El Liaison Committee on Medical Education (LCME), la organización relacionada con la acreditación de las escuelas de medicina en los Estados y en Canadá, ha establecido la pertinencia en el sentido de que los estudiantes de medicina deben reconocer los principios éticos y científicos básicos de la investigación científica y traslacional incluyendo la forma como esta investigación es realizada, analizada, y aplicada para la atención de las personas (Liaison Committee on Medical Education, 2011).

Durante un período de 6 años (2004 a 2009), en un estudio que incluyó a 562 estudiantes, se verificaron diferencias estadísticamente significativas sobre la percepción positiva en el post-test, comparado al ingreso en el pre-test, de la importancia de la inclusión de un curso de ciencias básicas en el cuarto año del currículum de los estudiantes de Medicina en la Universidad de Rochester en los Estados Unidos (DiGiovanni, Ward, \& Lambert, 2011).

A pesar de los avances en las diferentes disciplinas de las ciencias básicas biomédicas, se observa una reducción en la participación de varias de estas disciplinas en los diferentes currículos universitarios. En los programas de medicina, que es donde más se ha estudiado esta situación, se observa que, en términos generales, a nivel mundial existe una tendencia hacia la reducción de las horas de la enseñanza en ciencias básicas en varias instituciones universitarias. Por ejemplo, en el caso de la neuroanatomía, un estudio realizado en 31 Facultades de Medicina de los Estados Unidos, permitió verificar que las horas dedicadas a la enseñanza de la neuroanatomía disminuyeron un $18 \%$ entre el 2002 y el 2009 (Drake, McBride, Lachman, \& Pawlina, 2009).
Hasta que no se tenga un conocimiento más claro sobre cómo los conocimientos adquiridos en los años de estudios preclínicos se utilizan en la práctica, los argumentos y justificaciones de las ciencias básicas en el currículum continuarán dependiendo en gran medida de las teorías educativas y de las ideologías (Hazelton, 2011). De otra parte, por ejemplo, en el caso de la fisioterapia, en relación con la información del impacto del aprendizaje basado en problemas, aunque existe una literatura emergente, la investigación existente se origina principalmente todavía de la investigación médica (Verheyden, Handgraaf, Demirci, \& Grüneberg, 2011), siendo deseable aumentar la investigación especialmente sobre el impacto de los diferentes procesos de enseñanza-aprendizaje en la medicina, en la fisioterapia y en general en la rehabilitación y en las ciencias de la salud.

En la educación médica se proponen 3 grandes objetivos de las ciencias básicas: 1) apoyar el desarrollo de competencias para el razonamiento clínico; 2) apoyar el análisis crítico de las intervenciones médicas y quirúrgicas, es decir, de la medicina basada en la evidencia y 3) desde el enfoque traslacional apoyar el análisis de los procesos para mejorar la salud, es decir, la asistencia sanitaria (Grande, 2009).

\section{DISCUSIÓN}

Es evidente que la medicina traslacional se encuentra todavía en el período embrionario. En ciencias de la salud para la implementación del enfoque traslacional, es deseable acelerar los procesos que permitan pasar los descubrimientos de las ciencias básicas a la práctica clínica (Zerhouni, 2003), considerar estrategias para gerenciar hacia el futuro las investigaciones a través de equipos multisciplinarios (McGartland y otros, 2010), la evolución de departamentos de investigación a centros de investigación interdisciplinarios, el entrenamiento para aprender a trabajar en equipos interdisciplinarios, el reconocimiento del enfoque traslacional como una disciplina pertinente a nivel investigativo, y que los recursos de información sean eficientemente compartidos (Zerhouni, 2012). La educación traslacional retoma el concepto de la integración de la investigación básica con la práctica desde una perspectiva interdisciplinaria, dado que el conocimiento disciplinario por si sólo ya no será suficiente para la resolución de los problemas en virtud de la complejidad encontrada en los mismos (Ferring, 2009). Una mayor colaboración entre los investigadores en ciencias básicas y los clínicos permitirán establecer nuevos puntos de referencia para la investigación multidisciplinaria e interdisciplinaria debiéndose fortalecer la investigación traslacional para de esta manera generar entornos más competitivos a nivel científico (Lim, 2005). La investigación traslacional debe integrar, entre otros, a los profesionales de la salud, los científicos, las universidades, las empresas, los comités éticos y de asesoramiento y las agencias reguladoras gubernamentales (Wu, Marincola, Liebman, \& Wang, 2013).

\section{CONCLUSIONES}

El reto, del presente y del futuro, es lograr la integración de las ciencias básicas con las áreas clínicas considerando naturalmente la participación de las diferentes ciencias sociales lo que brindará un contexto educativo de mayor aplicabilidad y empleabilidad para los 
3 futuros profesionales. Por ejemplo, la integración del modelo biopsicosocial en un contexto interdisciplinario y multisciplinario hace parte de las competencias en la formación de fisioterapeutas que puedan ser profesionales éticos, críticos y reflexivos (Verheyden, Handgraaf, Demirci, \& Grüneberg, 2011) y que puedan desenvolverse en los diferentes situaciones inter-culturales que su práctica cotidiana lo requiera (Bialocerkowski, Wells, \& Grimmer-Somers, 2011), siendo este enfoque extensivo naturalmente a las diferentes disciplinas relacionadas con la rehabilitación y en general con las ciencias de la salud. Claramente debemos re-establecer las conexiones de un conocimiento biomédico y de una práctica que con el paso del tiempo se ha ido separando en ciencias básicas y en áreas clínicas con fronteras no siempre bien delimitadas aunque sí muy amplias lo cual no tiene razón de ser. La investigación relacionada con la educación traslacional abre una nueva frontera del conocimiento.

Ludwig Edinger (1855-1918), fundador de la neuroanatomía moderna, y citado al inicio de este artículo, tenía una profunda pasión por la investigación y por la enseñanza. Edinger enfocaba su interés en el estudio de la biología evolutiva, de la microscopía, de la ana- tomía macroscópica del sistema nervioso e incluso de la filosofía. Sumado a esto se encuentra su espíritu emprendedor al fundar, con sus propios medios, el que hoy es el Instituto Neurológico de la Universidad de Goethe en Frankfurt, siendo el Instituto Neurológico más antiguo de Alemania. Gnosis y Praxis son términos que él introdujo (Prithishkumar, 2011). Sin duda, se requerirán de varias de las cualidades de Edinger en las presentes y en las futuras generaciones de educadores y científicos para afrontar los diferentes retos de la educación superior en el área de la salud.

\section{AGRADECIMIENTOS}

Mis más sinceros agradecimientos por la revisión del presente artículo a los siguientes Profesores del Departamento de Ciencias Básicas de la Escuela Colombiana de Rehabilitación: Ana Lucía Cely Amézquita, Mg. en Desarrollo Educativo y Social; Oscar Ramos Combariza, Mg. en Gestión de Organizaciones; Wiliiam Hernández, Lic. En Biología y Química y a David Camilo Durán Chaparro, Ing. Mecánico. 


\section{Referencia:}

\section{Las referencias a otras obras son una parte muy importante en la literatura científica; ya que estas permiten conocer más sobre los autores y mantener vivas sus voces dentro del texto.}

Bandiera, G., Boucher, A., Neville, A., Kuper, A., \& Hodges, B. (2013). Integration and timing of basic and clinical sciences education. Med Teach. $35(5), 381-387$

Berthier, M. L., \& Pulvermüller, F. (2011). Neuroscience insights improve neurorehabilitation of poststroke aphasia. Nat Rev Neurol. , 7 (2), 86-97.

Bialocerkowski, A., Wells, C., \& Grimmer-Somers, K. (2011). Teaching physiotherapy skills in culturally-diverse classes. BMC Medical Education (11), 34 .

Buckingham, J. C. (2012). Integrating pharmacology and clinical pharmacology in universities. British Journal of Clinical Pharmacology, 73 (6), 861-865.

Burgoyne, L. N., O'Flynn, S., \& Boylan, G. B. (2010). Undergraduate medical research: the student perspective. Retrieved June 19, 2013, from Medical Education Online: http://hinari-gw.who.int/whalecomwww.ncbi.nlm.nih.gov/ whalecomo/pmc/articles/PMC2939395/\#!po=28.9474

Cabieses, B., \& Espinoza, M. A. (2011). La investigación traslacional y su aporte para la toma de decisiones en políticas de salud. Revista Peruana de Medicina Experimental y Salud Pública, 28 (2), 288-297.

Clark, F., Park, D. J., \& Burke, J. P. (2013). Dissemination: bringing translational research to completion. Am J Occup Ther. , 67 (2), 185-193.

Contopoulos-Ioannidis D.G., N. E. (2003). Translation of highly promising basic science research into clinical applications. Am J Med (114), 477-484 . .

Diab, A. A., \& Moustafa, I. M. (2012). Lumbar lordosis rehabilitation for pain and lumbar segmental motion in chronic mechanical low back pain: a randomized trial. J Manipulative Physiol Ther. , 35 (4), 246-253.

DiGiovanni, B. F., Ward, D. S., \& Lambert, D. (2011). Process of discovery: A fourth-year translational science course. Retrieved June 19, 2013, from http://hinari-gw.who.int/whalecomwww.ncbi.nlm.nih.gov/whalecomo/ pmc/articles/PMC3230242/

Drake, R., McBride, J. M., Lachman, N., \& Pawlina, W. (2009). Medical education in the anatomical sciences: The winds of change continue to blow. Anatomical Sciences Education (2), 253-259.
Ferrell, C. B. (2009). Reengineering clinical research science: a focus on translational research. Behav Modif, 33 (1), 7-23.

Ferring, D. (2009, June). Bridging education and training in ageing and disabilities: towards translational education. Retrieved June 17, 2013, from International Journal of Integrated Care: http://www.ijic.org/index.php/ijic/ article/view/URN:NBN:NL:UI:10-1-100584/682

Floel, A., \& Cohen, L. G. (2006). Translational studies in neurorehabilitation: from bench to bedside. Cogn Behav Neurol., 19 (1), 1-10.

Fuentes, A. (2009). Medicina traslacional en la oncología mexicana. GAMO, 8 (3), 81 .

Gamulin, S. (2003). Impact of molecular medicine on pathophysiology, medical practice, and medical education. Croat Med J., 44 (4), 374-385.

Grande, J. P. (2009). Training of physicians for the twenty-first century: role of the basic sciences. Med Teach., 31 (9), 802-806.

Hasselbart, C. (2008). Futuro de la Medicina traslacional en cáncer. Cancerología (4), 7-8.

Hazelton, L. (2011). Changing Concepts of Neuroanatomy Teaching in Medical Education. Teaching and Learning in Medicine , 23 (4), 359-364.

Holden, K. R., Cooper, S. L., \& Wong, J. G. (2012). Neuroscience curriculum changes and outcomes: medical university of South Carolina, 2006 to 2010. Neurologist., 18 (4), 190-195.

Ifejika-Jones, N. L., \& Barrett, A. M. (2011). Rehabilitation--emerging technologies, innovative therapies, and future objectives. Neurotherapeutics., $8(3), 452-462$.

Ioannidis, J. P. (2004). Materializing research promises: opportunities, priorities and conflicts in translational medicine. J Transl Med , 2 (1), 5.

Jones, E. G. (2007). Neuroanatomy: Cajal and after Cajal. Brain Res Rev, 55 $(2), 248-255$.

REV. COL. REH || Bogotá, Colombia || Volumen 12 || Páginas 22 - 30 || ISSN 1692 - 1879 
Kiefe, C. (2011, May 20). What is Translational Research? Retrieved June 19, 2013, from University of Massachusetts Medical School, Dept of Quantitative Health Sciences : http://escholarship.umassmed.edu/cgi/viewcontent. cgi?article $=1000 \&$ context $=$ cts_retreat\&sei-redir $=1 \&$ referer $=$ http $\% 3 \mathrm{~A} \% 2 \mathrm{~F} \%$ 2Fwww.google.com\%2Furl\%3Fsa\%3Dt\%26rct\%3Dj\%26q\%3Dtranslational\%252ot1\%252ot2\%252ot3\%2520t4\%26source\%3Dweb\%26cd\%3D 5\%26ved\%3DoCEgQFjAE\%26url\%

Lewis, H. P. (1956). Integration of basic science with clinical training. JAMA , $161(1), 27-29$.

Liaison Committee on Medical Education. (2011, May). Functions and structure of a medical school. Standards for Accreditation of Medical Education Programs Leading to the M.D. Degree. Retrieved June 19, 2013, from http://www. lcme.org/functions2o11may.pdf

Lifshitz, A. (2009). Medicina traslacional (traduccional, traducida, traslativa, trasladada). Med Int Mex, 25 (4), 251-253.

Lim, P. (2005). Translational Research. Annals Academy of Medicine, 34 (7), 409-410.

Mainous, A. G., Johnson, S. P., Chirina, S., \& Baker, R. (2013). Academic family physicians' perception of genetic testing and integration into practice: a CERA study. Fam Med. , 45 (4), 257-262.

McCormack, P., Southern, K. W., \& McNamara, P. S. (2012). New nebulizer technology to monitor adherence and nebulizer performance in cystic fibrosis. J Aerosol Med Pulm Drug Deliv., 25 (6), 307-309.

McGartland, D., Schoenbaum, E. E., Lee, L. S., Schteingart, D. E., Marantz, ,. P., Anderson, K. E., et al. (2010). Defining Translational Research: Implications for Training. Acad Med, 85 (3), 470-475.

Moon, K., Andreas, K., \& Cohen, A. R. (2010). The Birth and Evolution of Neuroscience Through Cadaveric Dissection. Neurosurgery, 67 (3), 799-810.

Overman, J. J., \& Carmichael, S. T. (2013, June 11). Plasticity in the Injured Brain : More than Molecules Matter. Retrieved June 16, 2013, from The Neuroscientist: http://nro.sagepub.com/content/early/2013/06/11/1073858413491146

Padilha, M. I. (2011). Translational research - what is its importance to nursing practice? Texto Contexto Enferm, 20 (3), 221-222.

Prithishkumar, I. J. (2011). Ludwig Edinger (1855-1918): Founder of Modern Neuroanatomy. Clinical Anatomy, 25 (2), 155-157.

Proudfoot, A. G., Hind, M., \& Griffiths, M. J. (2011). Biomarkers of acute lung injury: worth their salt? Retrieved June 19, 2013, from http://hinari-gw.who.int/whalecomwww.ncbi.nlm.nih.gov/whalecomo/pmc/articles/ $\mathrm{PMC}_{3261814 /}$

Ramjiawan, B., Pierce, G. N., Kabir Anindo, M. I., AlKukhun4, A., Alshammari, A., Chamsi, A. T., et al. (2012). An international basic science and clinical research summer program for medical students. Adv Physiol Educ (36), 27-33.

REV. COL. REH || Bogotá, Colombia || Volumen 12 || Páginas 22 - 30 || ISSN 1692 - 1879
Raymer, A. M., Beeson, P., Holland, A., Kendall, D., Maher, L. M., Martin, N., et al. (2008). Translational research in aphasia: from neuroscience to neurorehabilitation. J Speech Lang Hear Res., 51 (1), 259-75.

Shuldiner, A. R., Relling, M. V., Peterson, J. F., Hicks, K., Freimuth, R. R., Sadee, W., et al. (2013, March 19). The Pharmacogenomics Research Network Translational Pharmacogenetics Program: Overcoming Challenges of Real-World Implementation. Retrieved June 16, 2013, from Nature.com.

Sitaraman, R. (2012). From bedside to blackboard: the benefits of teaching molecular biology within a medical context. Perspect Biol Med., 55 (3), 461-466.

Smeesters, P. R., Deghorain, M., \& Steer, A. C. (2011). Science that "knows" and science that "asks". Journal of Translational Medicine, 9, 128.

Steiner, M. C., Roubenoff, R., Tal-Singer, R., \& Polkey, M. I. (2012). Prospects for the development of effective pharmacotherapy targeted at the skeletal muscles in chronic obstructive pulmonary disease: a translational review. Thorax., 67 (12), 1102-1109.

Sullivan, K. (2010). Translational Rehabilitation Research: From Science to Practice and Practice to Science. Journal of Neurologic Physical Therapy, $34(2), 119-121$.

Tworek, J. K., Jamniczky, H. A., Jacob, C., \& Hallgrímsson, B. (2013). The LINDSAY virtual human project: an immersive approach to anatomy and physiology. Anat Sci Educ., 6 (1), 19-28.

Verheyden, G., Handgraaf, M., Demirci, A., \& Grüneberg, C. (2011). The future of physiotherapy education: Towards a translational model of learning complex skills. Physiotherapy Research International, 16 (4), 187-190.

Weber, G. M. (2013). Identifying translational science within the triangle of biomedicine. Journal of Translational Medicine (11), 126.

Wilcox, J. T., Cadotte, D., \& Fehlings, M. G. (2012). Spinal cord clinical trials and the role for bioengineering. Neurosci Lett., 519 (2), 93-102.

Woolf, S. H. (2008). The Meaning of Translational Research and Why It Matters. JAMA, 299 (2), 211-213.

Wu, X., Marincola, F. M., Liebman, M. N., \& Wang, X. (2013). A global resource to translational medicine: the International Park of Translational Medicine and BioMedicine (IPTBM). Journal of Translational Medicine (11), 8.

Zerhouni, E. A. (2012, June). Trends and challenges in translational research. ED MILLER SYMPOSIUM, JOHNS HOPKINS. Retrieved June 17, 2013, from http://web.jhu.edu/administration/provost/initiatives/Zerhouni.pdf

Zerhouni, E. (2003). Medicine: The NIH Roadmap. Science (302), 63-72.

Zinchuk, A. V., Flanagan, E. P., Tubridy, N. J., Miller, W. A., \& McCullough, L. D. (2010). Attitudes of US medical trainees towards neurology education: "Neurophobia" - a global issue. BMC Med Educ. (10), 49. 